\title{
Year 1 of the ZTF high-cadence Galactic plane survey: strategy, goals, and early results on new single-mode hot subdwarf B-star pulsatos
}

Thomas Kupfer, ${ }^{1,2 \star}$ Thomas A. Prince ${ }^{\circledR},{ }^{3}$ Jan van Roestel, ${ }^{3}$ Eric C. Bellm, ${ }^{4}$ Lars Bildsten, ${ }^{5,1}$ Michael W. Coughlin ${ }^{\circledR}, 6$ Andrew J. Drake, ${ }^{3}$ Matthew J. Graham ${ }^{\circledR},{ }^{3}$ Courtney Klein ${ }^{\circledR}$, Shrinivas R. Kulkarni, ${ }^{3}$ Frank J. Masci, ${ }^{8}$ Richard Walters, ${ }^{9}$ Igor Andreoni, ${ }^{3}$ Rahul Biswas ${ }^{(1)}, 10$ Corey Bradshaw, ${ }^{2}$ Dmitry A. Duev ${ }^{\circ}, 3$ Richard Dekany, ${ }^{9}$ Joseph A. Guidry, ${ }^{11}$ J. J. Hermes, ${ }^{12}$ Russ R. Laher ${ }^{8}$ and Reed Riddle ${ }^{(1) 9}$

${ }^{1}$ Kavli Institute for Theoretical Physics, University of California, Santa Barbara, CA 93106, USA

${ }^{2}$ Department of Physics and Astronomy, Texas Tech University, PO Box 41051, Lubbock, TX 79409, USA

${ }^{3}$ Division of Physics, Mathematics and Astronomy, California Institute of Technology, Pasadena, CA 91125, USA

${ }^{4}$ DIRAC Institute, Department of Astronomy, University of Washington, 3910 15th Avenue NE, Seattle, WA 98195, USA

${ }^{5}$ Department of Physics, University of California, Santa Barbara, CA 93106, USA

${ }^{6}$ School of Physics and Astronomy, University of Minnesota, Minneapolis, MN 55455, USA

${ }^{7}$ Department of Physics and Astronomy, University of California, Irvine, CA 92697, USA

${ }^{8}$ IPAC, California Institute of Technology, 1200 E. California Blvd, Pasadena, CA 91125, USA

${ }^{9}$ Caltech Optical Observatories, California Institute of Technology, Pasadena, CA 91125, USA

${ }^{10}$ Department of Physics, The Oskar Klein Center, Stockholm University, AlbaNova, SE-10691 Stockholm, Sweden

${ }^{11}$ Department of Astronomy, The University of Texas at Austin, Austin, TX 78712, USA

${ }^{12}$ Department of Astronomy, Boston University, 725 Commonwealth Ave., Boston, MA 02215, USA

Accepted 2021 May 5. Received 2021 May 5; in original form 2021 January 14

\begin{abstract}
We present the goals, strategy, and first results of the high-cadence Galactic plane survey using the Zwicky Transient Facility (ZTF). The goal of the survey is to unveil the Galactic population of short-period variable stars, including short-period binaries, and stellar pulsators with periods less than a few hours. Between 2018 June and 2019 January, we observed 64 ZTF fields resulting in $2990 \mathrm{deg}^{2}$ of high stellar density in the ZTF- $r$ band along the Galactic plane. Each field was observed continuously for 1.5 to $6 \mathrm{~h}$ with a cadence of $40 \mathrm{sec}$. Most fields have between 200 and 400 observations obtained over 2-3 continuous nights. As part of this survey, we extract a total of $\approx 230$ million individual objects with at least 80 epochs obtained during the high-cadence Galactic plane survey reaching an average depth of ZTF- $r \approx 20.5$ mag. For four selected fields with 2-10 million individual objects per field, we calculate different variability statistics and find that $\approx 1-2$ per cent of the objects are astrophysically variable over the observed period. We present a progress report on recent discoveries, including a new class of compact pulsators, the first members of a new class of Roche lobe filling hot subdwarf binaries as well as new ultracompact double white dwarfs and flaring stars. Finally, we present a sample of 12 new single-mode hot subdwarf B-star pulsators with pulsation amplitudes between $\mathrm{ZTF}-r=20-76 \mathrm{mmag}$ and pulsation periods between $P=5.8-16 \mathrm{~min}$ with a strong cluster of systems with periods $\approx 6 \mathrm{~min}$. All of the data have now been released in either ZTF Data Release 3 or Data Release 4.
\end{abstract}

Key words: surveys - binaries (including multiple): close - stars: oscillations (including pulsations)-white dwarfs.

\section{INTRODUCTION}

Large-scale optical time-domain surveys have opened a new window to study the variable sky providing hundreds to thousands of epochs across the whole sky. Starting with the Sloan Digital Sky Survey (SDSS; York et al. 2000), a new generation of wide-field optical surveys has exploited new affordable CCD detectors to open the frontier of data-intensive astronomy. This allows the study of stellar variability on different time-scales across the full magnitude range. In particular, surveys covering also low Galactic latitudes are well suited to study the Galactic distribution of photometric variable stars.

^E-mail: tkupfer@ttu.edu
Ground-based surveys include the Optical Gravitational Lensing Experiment (OGLE; e.g. Soszyński et al. 2015), PTF (Law et al. 2009), the Vista Variables in the Via Lactea (; Saito et al. 2012), ASAS-SN, (Shappee et al. 2014; Jayasinghe et al. 2018), and most recently ATLAS (Heinze et al. 2018; Tonry et al. 2018). In particular, the fastest stellar variabilities on time-scales of minutes to hours are of great interest for a large number of scientific questions. This includes ultracompact binaries (UCBs), compact pulsators as well as fast flaring stars.

UCBs are a class of binary stars with orbital periods less than about $60 \mathrm{~min}$, consisting of a neutron star (NS)/white dwarf (WD) primary and a Helium-star (He-star)/WD/NS secondary. These UCBs are sources of low-frequency gravitational wave signals as probed 
by the Laser Interferometer Space Antenna (LISA) and are crucial to our understanding of compact binary evolution and offer pathways towards Type Ia and other thermonuclear supernovae. Systems with orbital periods $<20$ min will be the strongest Galactic LISA sources and will be detected by LISA within weeks after its operation begins (Nelemans, Yungelson \& Portegies Zwart 2004; Ströer \& Vecchio 2006; Nissanke et al. 2012; Littenberg et al. 2013; Korol et al. 2017; Kremer et al. 2017; Kupfer et al. 2018; Lamberts et al. 2019; Burdge et al. 2019, 2020a) and as such are ideal multimessenger sources (Shah, van der Sluys \& Nelemans 2012; Shah \& Nelemans 2014; Baker et al. 2019; Littenberg et al. 2019; Kupfer et al. 2019a)

Short time-scale photometric variations can also originate from astrophysical changes within the internal structure or atmosphere of the star. Such sources are flaring stars, pulsating stars, or white dwarfs. Prominent examples of rapidly pulsating stars are $\delta$ Scuti stars (Breger 2000), SX Phoenicis variables (Nemec \& Mateo 1990), or Ap or Am stars (Renson, Gerbaldi \& Catalano 1991), and pulsating white dwarfs (ZZ Ceti variables; Fontaine \& Brassard 2008) as well as pulsating extremely low-mass white dwarfs (Hermes et al. 2013). The stars exhibit pulsation amplitudes of less than 1 percent up to several tens of percent on time-scales of few to tens of minutes.

Recently, a new class of short-period pulsating hot stars known as Blue Large-Amplitude Pulsators (BLAPs) was discovered by Pietrukowicz et al. (2017). Their pulsation periods are typically between 20-40 min. Romero et al. (2018) and Byrne \& Jeffery (2018) proposed that the BLAPs are hot pre-helium white dwarfs that are cooling and contracting, with masses in the range $0.3-0.35 \mathrm{M}_{\odot}$. A recent study by Meng et al. (2020) suggests that BLAPs could be the surviving companions of type Ia supernovae. In this scenario mass transfer from the BLAP progenitor leads to the detonation of a white dwarf companion, leaving behind a BLAP as single star. Their pulsation properties can best be explained by fundamental radial mode pulsators. However, the known sample of BLAPs is small and in-homogeneous, discovered only in the OGLE survey.

A number of fast cadence ground-based surveys have been executed to study the variable sky down to a few minutes period. The main goal of these high-cadence surveys is the discovery of rapid brightness variations seen in UCBs, compact pulsators as well as fast flaring stars. The first survey at low Galactic latitudes targeting shortperiod systems was the Rapid Temporal Survey (RATS; Ramsay \& Hakala 2005; Barclay et al. 2011) covering a total of $46 \mathrm{deg}^{2}$. Another more recent survey is the OmegaWhite (OW) survey, which covers a total of $400 \mathrm{deg}^{2}$ at low Galactic latitudes $\left(|b|<10^{\circ}\right)$ as well as in the Galactic Bulge using high-cadence optical observations. Two neighbouring $1 \mathrm{deg}^{2}$ fields are alternatingly observed in 39-s exposures over an observing duration of $2 \mathrm{~h}$, with an observational median cadence of $\approx 2.7 \mathrm{~min}$ per field (Macfarlane et al. 2015, 2017a,b; Toma et al. 2016; Kupfer et al. 2017)

As part of the Zwicky Transient Facility (ZTF), the Palomar 48inch (P48) telescope images the sky every clear night conducting several surveys including a Northern Sky Survey with a 3-d cadence, as well as smaller surveys such as a 1-d Galactic plane survey and simultaneous observations of the Northern TESS sectors (Graham et al. 2019; van Roestel et al. 2019; Bellm et al. 2019a,b). As part of the partnership share of ZTF, we conducted a dedicated high-cadence Galactic plane survey with a cadence of $40 \mathrm{sec}$ at low Galactic latitudes aiming to find UCBs and compact pulsators. During that dedicated survey, we either observed one field or alternated between two adjacent fields continuously for $\approx 1.5-3 \mathrm{~h}$ on two to three consecutive nights in the ZTF- $r$ band. Here, we present an overview of the ZTF high-cadence Galactic plane survey executed in ZTF year 1 . We present the observing strategy as well as some survey statistics.
Table 1. Overview of the high-cadence Galactic plane survey.

\begin{tabular}{lccc}
\hline Period & No. of fields & $\begin{array}{r}\text { Sky coverage } \\
\left(\mathrm{deg}^{2}\right)\end{array}$ & Filter \\
\hline $06-15-2018-07-31-2018$ & 16 & 750 & ZTF- $r$ \\
$08-03-2018-08-18-2018$ & 14 & 650 & ZTF- $r$ \\
$11-15-2018-01-15-2019$ & 34 & 1590 & ZTF- $r$ \\
\hline
\end{tabular}

We show a progress report and finalize with some early results from the survey. In Section 2, we discuss the observing strategy as well as the field selection and the data processing. In Section 3, we present results for four representative fields and in Section 4 we give a progress report of already published results. In Section 5, we show some new early results from the high-cadence Galactic plane survey and summarize and conclude in Section 6.

\section{DESIGN OF THE HIGH-CADENCE GALACTIC PLANE SURVEY}

ZTF uses a $47 \mathrm{deg}^{2}$ camera consisting of 16 individual CCDs each $6 \mathrm{k}$ $\times 6 \mathrm{k}$ covering the full focal plane of the $\mathrm{P} 48$ telescope. The ZTF highcadence Galactic plane survey covered a total of $\approx 2990 \mathrm{deg}^{2}$ split in 64 individual ZTF fields observed over three observing blocks; mid-June-July 2018, two weeks in 2018 August and November 15 to 2019 January 15. All observations of the high-cadence Galactic plane survey were obtained in ZTF- $r$ band. Each field has $\approx 200$ 400 epochs. The images were processed using ZTF data-processing pipeline described in full detail in Masci et al. (2019).

During June and July, we observed 16 fields covering $\approx 750 \mathrm{deg}^{2}$ mostly at low Galactic longitudes. In June, we observed every field continuously for $1 \mathrm{~h} 15 \mathrm{~min}$ and in July for $1 \mathrm{~h} 25 \mathrm{~min}$. All fields were observed over 2 or 3 consecutive nights. In August, 14 fields covering $\approx 650 \mathrm{deg}^{2}$ were observed over two weeks (see Table 1 ). We alternated between two adjacent fields continuously for $2 \mathrm{~h}$ 40min each night. The same fields were repeated the following night. The observations in June/July and August were done under stable conditions with an average seeing of $\approx 2$ arcsec. We lost only a total of five nights due to weather during June/July and August observations.

Between 2018 November 15 and 2019 January 15 high-cadence Galactic plane observations were scheduled for every night. We observed an additional 33 fields covering $\approx 1550 \mathrm{deg}^{2}$ with stable weather conditions (see Table 1). The overall strategy varied during those two months due to unstable weather. Most fields were observed alternating with adjacent fields but in particular low declination fields were observed continuously. Because more time was available each night most fields were observed for $\approx 3 \mathrm{~h}$. However, due to the unstable weather conditions about half of the fields were only observed in a single night and not repeated in subsequent nights. All other fields were observed over 2 or 3 nights. Although the seeing varies strongly between 1.7 and 4 arcsec, each field has a limiting magnitude of $>19.5 \mathrm{mag}$. A detailed overview of the fields observed in stable weather conditions is given in Tables A1-A3.

\subsection{Field selection}

The main science driver for the survey is to find and study UCBs consisting of fully degenerate or semi-degenerate stars. Hence, we selected ZTF fields based on the density of objects residing well below the main sequence, including mostly white dwarfs and hot subdwarf stars. 


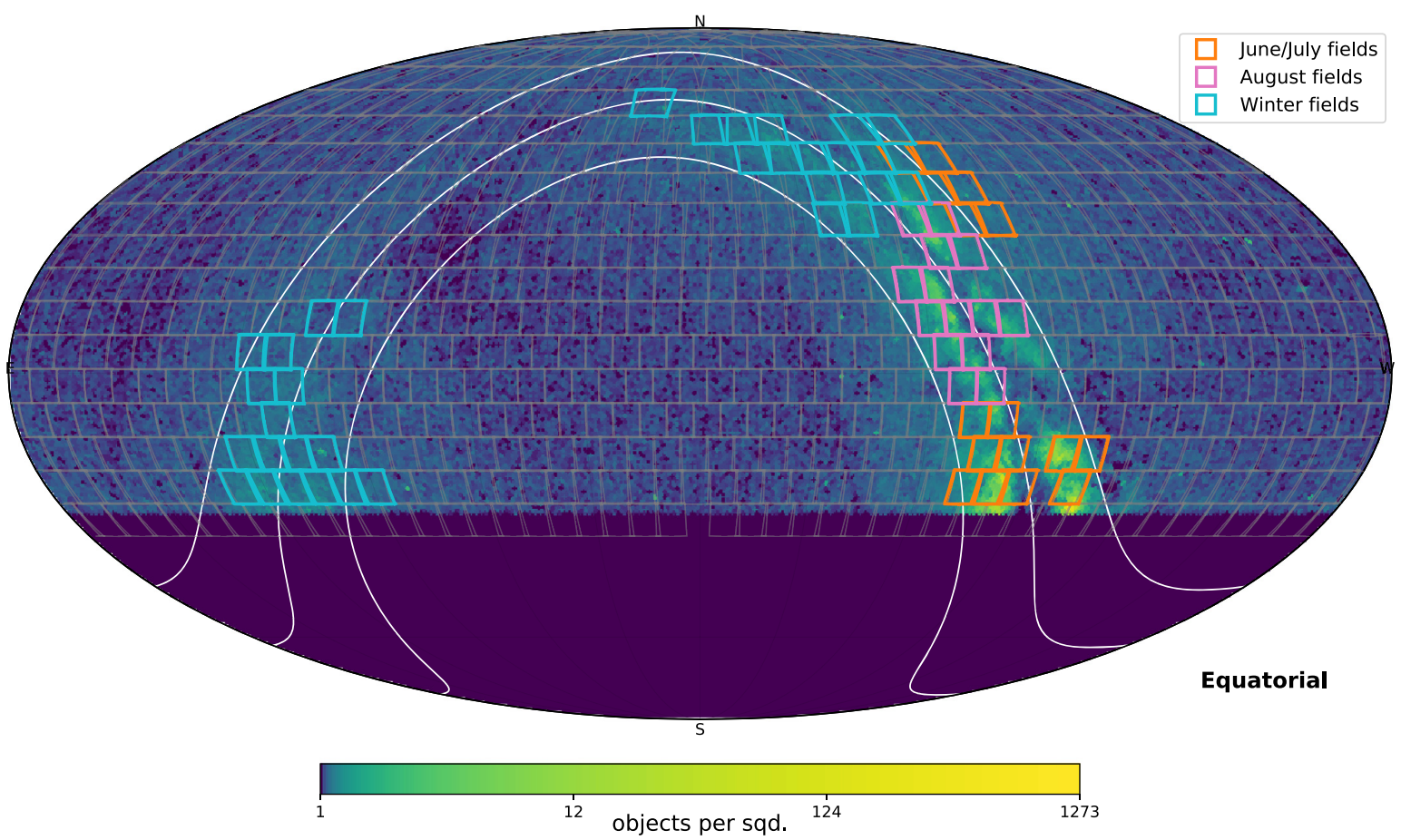

Figure 1. Sky density of candidate white dwarfs and hot subdwarfs selected from Gaia DR2 with the selected fields of the ZTF high-cadence Galactic plane survey observed in ZTF year-1. The squares show individual ZTF fields which have been observed in high-cadence Galactic plane observations. The white lines correspond to the Galactic equator and $|b|=15^{\circ}$.

To achieve this, we extracted objects with absolute magnitudes which placed them below the main-sequence based on Gaia data release 2 (Gaia Collaboration et al. 2016, 2018). Only objects with declinations $>-30^{\circ}$ were selected. As the goal was not to extract a clean sample we used a very relaxed tolerance for the parallax precision $\left(\varpi / \sigma_{\varpi}>3\right)$ and did not include any additional quality cuts, resulting in $\approx 350000$ individual objects (Fig. 2). For each object, the ZTF field was calculated and the fields with the largest number of individual objects were selected for our survey. Selected fields with the highest density have $\approx 1000$ selected objects per $\mathrm{deg}^{2}$. The final field selection was mainly driven by stellar density but also included other aspects like visibility and weather. Fig. 1 shows the sky density of the selected sources overplotted with the fields observed as part of the highcadence Galactic plane survey. As the stellar density is highest at low Galactic latitudes most fields are located at Galactic latitudes $\leq 15^{\circ}$.

\subsection{Data-processing and light-curve extraction}

Data-processing and light-curve generation follows the standard procedure for ZTF and occurs at the Infrared Processing and Analysis Center, Caltech. The raw camera image data are first instrumentally calibrated and astrometric solutions are derived using the Gaia DR1 catalogue. Sources are detected and fluxes measured using both aperture (Bertin \& Arnouts 1996) and PSF-fit photometry (Stetson 1987). Sources are photometrically calibrated using the Pan-STARRS1 DR1 catalogue. The epochal image data are then co-added within their respective survey fields and camera readout channels to construct reference images. Each reference image is constructed using a minimum of 15 and maximum of 40 good-quality epochal images, yielding depths of $\approx 2-2.5$ mag deeper than the single-epoch images.

The reference image co-adds are archived for use in other downstream processing: image differencing and light curve construction. To support light curve generation, sources are first detected and

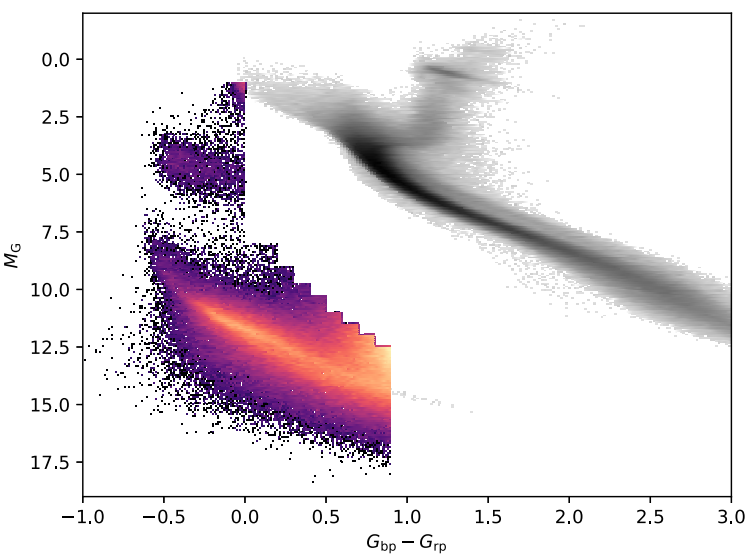

Figure 2. Hertzsprung-Russell diagram of the objects used for the field selection. The grey shaded region corresponds to the underlying HertzsprungRussell diagram showing the position of the main sequence and the red giant branch. The colour-coded region corresponds to the objects which were selected for the field selection. The clump around $M_{\mathrm{G}} \approx 5$ corresponds to the hot subdwarfs whereas the region below corresponds to the white dwarfs.

extracted from each reference image using PSF-fit photometry by running the DAOPhot utility (Stetson 1987). These sources provide the seeds to facilitate positional cross-matching across all the single-epoch-based PSF extraction catalogues going back to the beginning of the survey. PSF-fitting on the single-epoch images is also performed using DAOPhot. These single-epoch images are direct single exposure images, not difference images. A fixed sourcematch radius of 1.5 arcsec is used for the positional matching. Only the PSF-fit-derived positions and photometry, along with selected PSF-fit metrics are retained during the source-matching process. Aperture photometry is not propagated to the light-curve metadata. 


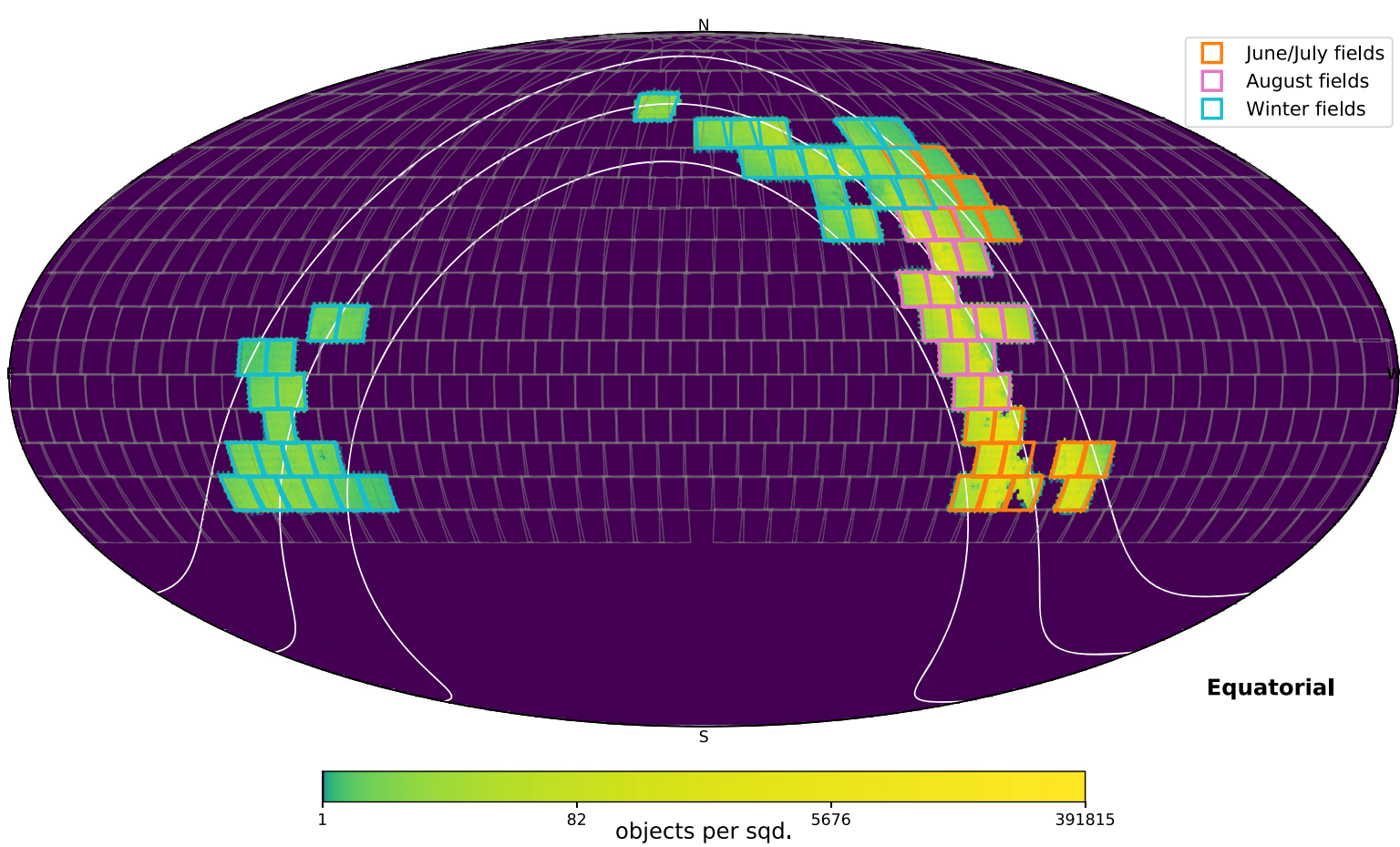

Figure 3. Sky density of individual objects with at least 80 epochs from the ZTF high-cadence Galactic plane survey observed in ZTF year-1. The squares show individual ZTF fields, which have been observed in high-cadence Galactic plane observations. The white lines correspond to the Galactic equator and $|b|=15^{\circ}$.

Further details of the data processing, PSF-fit photometry software, light-curve generation, formats, and overall performance on photometric accuracy are described in Masci et al. (2019). We extracted the light curves from each object which has at least 80 epochs obtained as part of the high-cadence Galactic plane survey from the ZTF light curve data base 'Kowalski' and extracted a total of $\approx 230$ million light curves across all observed fields. Fig. 3 shows the sky density of the extracted objects overplotted with the fields observed as part of the high-cadence Galactic plane survey.

\section{RESULTS FROM REPRESENTATIVE FIELDS}

We select four representative fields with different stellar densities and calculate statistics for each individual light curve, including median magnitude, reduced $\chi^{2}$, interquartile range (IQR), skewness, inverse von-Neumann, Stetson J, and Stetson K statistics. We refer to table 2 in Coughlin et al. (2020) for a detailed description of the individual statistics. These statistics are used to evaluate light curve variability and identify variable objects in the high-cadence Galactic plane data.

The selected fields are FieldID $=331(l=8.59, b=8.69)$, FieldID $=538 \quad(l=44.64, b=3.09), \quad$ FieldID $=769$ $((l=91.87, b=2.38) l=91.87 \mathrm{deg}, b=-2.38 \mathrm{deg})$ and FieldID $=$ $309(l=230.68, b=2.98)$. Fig. 4 shows the stellar density of the four selected fields. The different colouring correspond to different densities. Some structure of lower and higher density regions can be seen in the fields. The white circles are masked regions due to saturated stars and the horizontal and vertical gaps are chip gaps between the 16 CCDs. The two white square areas in FieldID 331 are individual quadrants, which were not processed. FieldID $=331$ was observed in three consecutive nights for $1.5 \mathrm{~h}$ each night. FieldID $=538$ was observed for two consecutive nights for 1.5 and $2.5 \mathrm{~h}$. FieldID $=769$ was observed for two consecutive nights for $3 \mathrm{~h}$ per night and FieldID $=309$ was observed for two consecutive nights for 1.7 and $3 \mathrm{~h}$. See Tables A1-A3 for more details. The four selected fields represent a large range of stellar densities from $\approx 10$ million individual light curves for FieldID $=$ 331, $\approx 5.8$ million for FieldID $=538, \approx 3.8$ million for FieldID $=$ 769 , and $\approx 2.3$ million for FieldID $=309$.

Different statistics can be used to evaluate whether an object is considered variable or not. We decided to use the IQR which corresponds to the difference between the upper and lower quartile values. The main advantage of IQR is that it is a robust statistic not affected by extreme outliers due to individual bad photometry data points and therefore a better estimate of the general spread around the median magnitude. When there are outliers in a sample, the median and IQR are best used to summarize a typical value and the variability in the sample, respectively. Large variability in the light curve leads to a larger IQR.

To estimate whether an object is variable in the high-cadence Galactic plane data, we used the following approach. We calculate the median ZTF- $r$ band magnitude for each object. The full magnitude range of all objects in a ZTF field is binned into several bins and each object is added to a bin based on its median magnitude. We used bin sizes of $0.5 \mathrm{mag}$ between 12 and $16.5 \mathrm{mag}$ where the IQR is constant at $\approx 0.02 \mathrm{mag}$ for non-variable sources. Above $16.5 \mathrm{mag}$, we used bin sizes of $0.2 \mathrm{mag}$ because the IQR for non-variable sources is increasing with increasing magnitudes to $\approx 0.2 \mathrm{mag}$ at the faint end at ZTF- $r=20.5 \mathrm{mag}$ because the underlying noise of the sources is increasing for fainter sources. Each bin contains at least a few thousand objects.

For each magnitude bin, we then calculate a histogram of IQR values. If there are only constant sources with a similar noise pattern, a normal distribution in an IQR histogram is expected. An excess of variable light curves will result in a deviation from a normal distribution towards more objects with larger IQR values. To estimate the excess and number of variable objects, we fit a Gaussian to each IQR histogram and define an object as variable if, in a given histogram bin, less than 5 per cent of the sources in that magnitude bin are 
Table 2. Photometric properties of the high-gravity-BLAPs.

\begin{tabular}{|c|c|c|c|c|c|c|c|c|}
\hline Object & $\begin{array}{c}\mathrm{RA}(\mathrm{J} 2000) \\
\left({ }^{h}:{ }^{\mathrm{min}}:{ }^{\mathrm{sec}}\right)\end{array}$ & $\begin{array}{c}\text { Dec. }(\mathrm{J} 2000) \\
\left({ }^{\circ}:^{\prime}:\right)\end{array}$ & $\begin{array}{l}P \\
\text { (s) }\end{array}$ & $\begin{array}{l}A_{\mathrm{ZTF}-\mathrm{r}}^{b} \\
\text { (mmag) }\end{array}$ & $\begin{array}{c}\varpi^{c} \\
(\mathrm{mas})\end{array}$ & $\begin{array}{c}g^{a} \\
(\mathrm{mag})\end{array}$ & $\begin{array}{l}g-r^{a} \\
(\mathrm{mag})\end{array}$ & FieldID \\
\hline ZTF-sdBV1 & 18:26:36.09 & $+10: 00: 22.3$ & $347.287 \pm 0.02$ & 19.9 & $0.5640 \pm 0.0372$ & 14.90 & -0.41 & 538 \\
\hline ZTF-sdBV2 & $19: 13: 54.77$ & $-11: 05: 19.4$ & $360.576 \pm 0.02$ & 19.6 & $0.4568 \pm 0.0888$ & 16.24 & -0.33 & 385 \\
\hline ZTF-sdBV3 & 21:03:08.50 & $+44: 4314.9$ & $365.822 \pm 0.01$ & 53.3 & $0.3550 \pm 0.0769$ & 16.07 & -0.31 & 768 \\
\hline ZTF-sdBV4 & 08:06:07.05 & $-20: 0819.1$ & $370.481 \pm 0.02$ & 26.0 & $0.4021 \pm 0.0531$ & 16.37 & -0.32 & 311 \\
\hline ZTF-sdBV5 & $21: 49: 45.56$ & $+45: 5836.5$ & $375.690 \pm 0.02$ & 36.1 & $0.3112 \pm 0.0683$ & 16.55 & -0.35 & 770 \\
\hline ZTF-sdBV6 & $07: 40: 00.71$ & $-14: 4202.5$ & $375.782 \pm 0.02$ & 21.1 & $0.5979 \pm 0.0394$ & 15.10 & -0.36 & 310 \\
\hline ZTF-sdBV7 & $19: 24: 08.62$ & $-12: 5830.9$ & $377.468 \pm 0.02$ & 19.3 & $0.7399 \pm 0.0355$ & 14.64 & -0.41 & 385 \\
\hline ZTF-sdBV8 & $06: 54: 48.55$ & $-25: 2208.3$ & $435.459 \pm 0.02$ & 31.4 & $0.5458 \pm 0.0318$ & 13.93 & -0.44 & 259 \\
\hline ZTF-sdBV9 & $18: 52: 49.07$ & $-18: 4608.4$ & $524.829 \pm 0.02$ & 73.4 & $0.1427 \pm 0.1538$ & 17.12 & -0.38 & 334 \\
\hline ZTF-sdBV10 & $19: 49: 59.34$ & $+08: 3106.1$ & $646.306 \pm 0.02$ & 32.8 & $0.3884 \pm 0.0642$ & 16.23 & -0.38 & 541 \\
\hline ZTF-sdBV11 & $07: 18: 43.69$ & $-02: 2931.2$ & $688.485 \pm 0.02$ & 30.2 & $0.1571 \pm 0.1329$ & 17.52 & -0.33 & 411 \\
\hline ZTF-sdBV12 & $17: 07: 41.34$ & $-15: 2242.9$ & $995.479 \pm 0.02$ & 37.3 & $0.4376 \pm 0.0346$ & 14.11 & -0.35 & 330 \\
\hline
\end{tabular}

${ }^{a}$ From the Pan-STARRS release 1 (PS1) survey (Chambers et al. 2016) reddening corrected using Green et al. (2019).

${ }^{b}$ Amplitude in ZTF- $r$ from the ZTF light curves.

${ }^{c}$ Parallaxes from Gaia EDR3 (Gaia Collaboration et al. 2016, 2020).
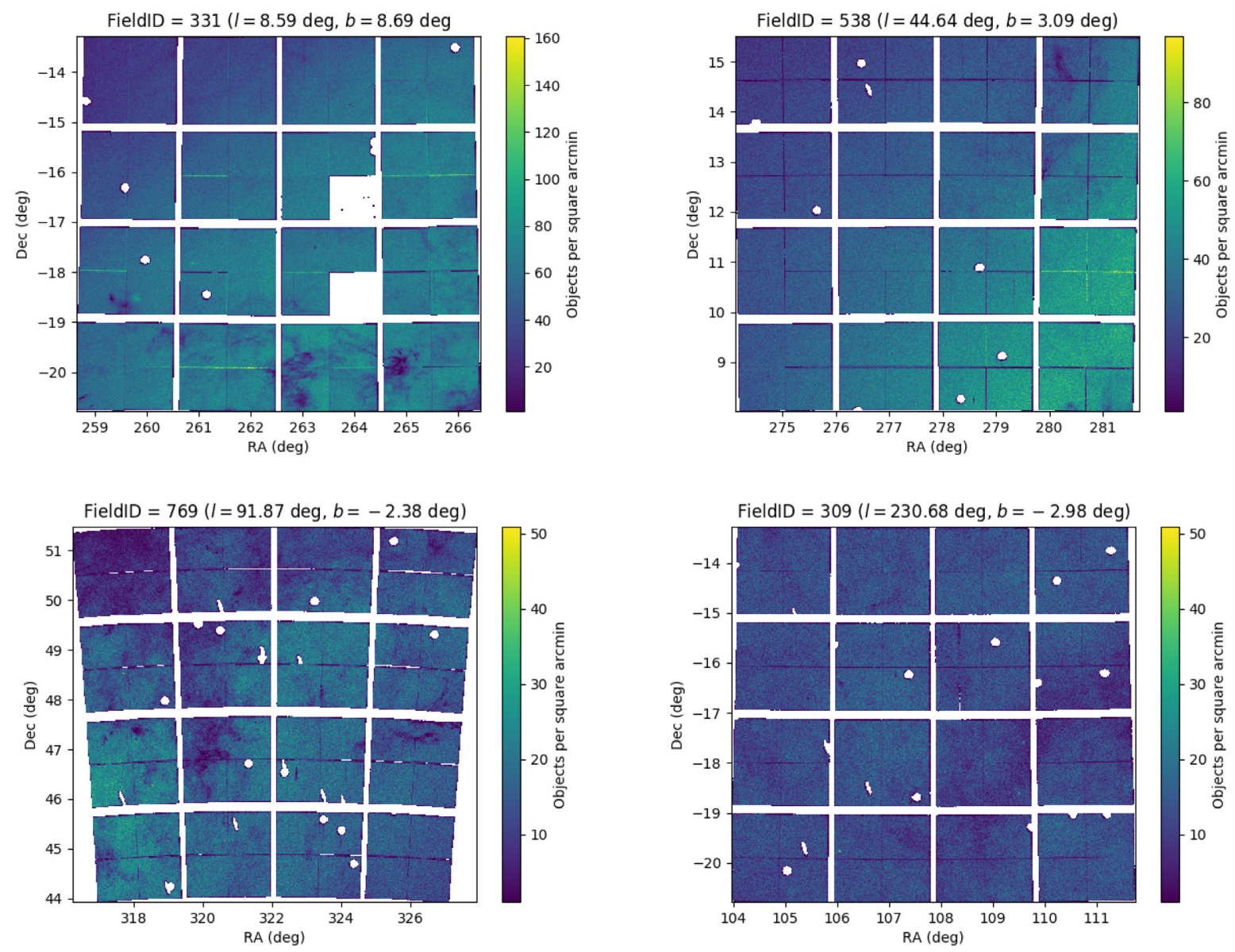

Figure 4. Sky density of ZTF objects with at least 80 epochs obtained during the high-cadence Galactic plane survey for four selected fields. The white circles are masked regions due to saturated stars and the horizontal and vertical gaps are chip gaps between the 16 CCDs. The two white square areas in FieldID 331 are individual quadrants, which were not processed.

considered constant based on the Gaussian fit. Fig. 5 shows two examples of IQR histograms with Gaussian fits of FieldID = 309 and 331 for the magnitude range $15.5-16 \mathrm{mag}$. The orange curve corresponds to the Gaussian fit of the underlying histogram and the red-dashed line corresponds to the IQR limit above which we call an object a variable.
Using this method, we find 15 per cent variable sources for FieldID $=331,9$ per cent variable sources for FieldID $=769$, and FieldID $=538$ and 5 per cent variable sources for FieldID $=309$ (see Fig. 6). The number of variable sources per field decreases with the stellar density of the field and it is likely that blending could produce false positives where the object appears variable due to a close-by 

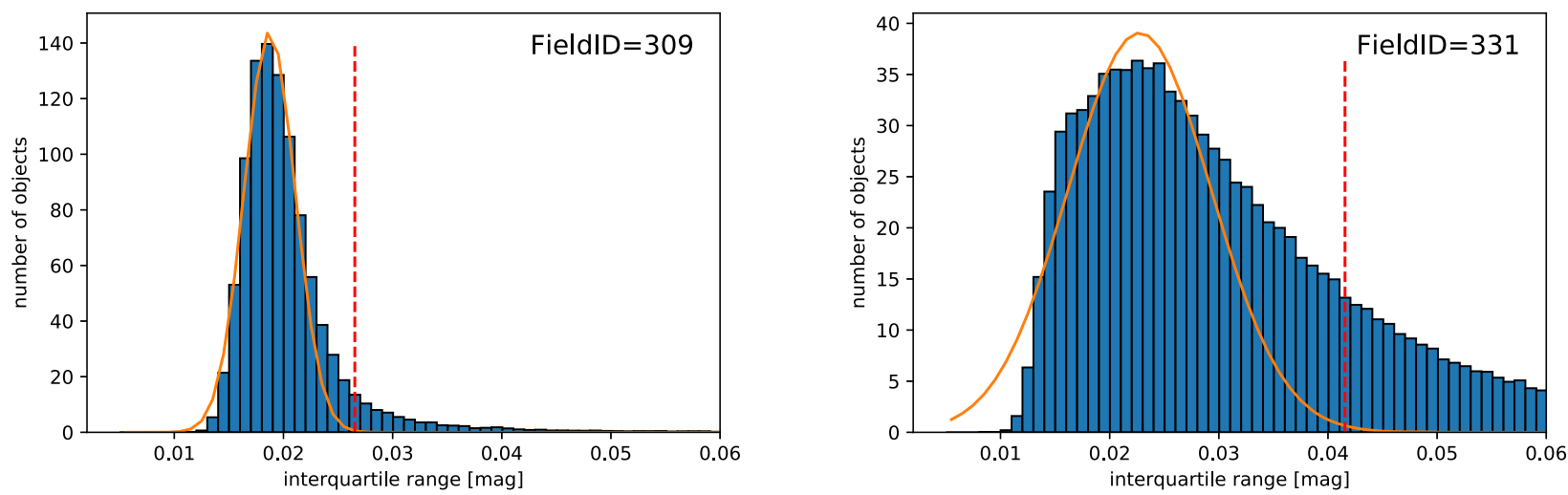

Figure 5. Histogram of the IQR extracted at a ZTF- $r$ median magnitude between 15.5 and 16 with the best Gaussian fit and the limit above which an object is called variable. The fit to the histogram in FieldID $=331$ is poor, which is likely due to a large number of blended sources. See section 3 for more details.
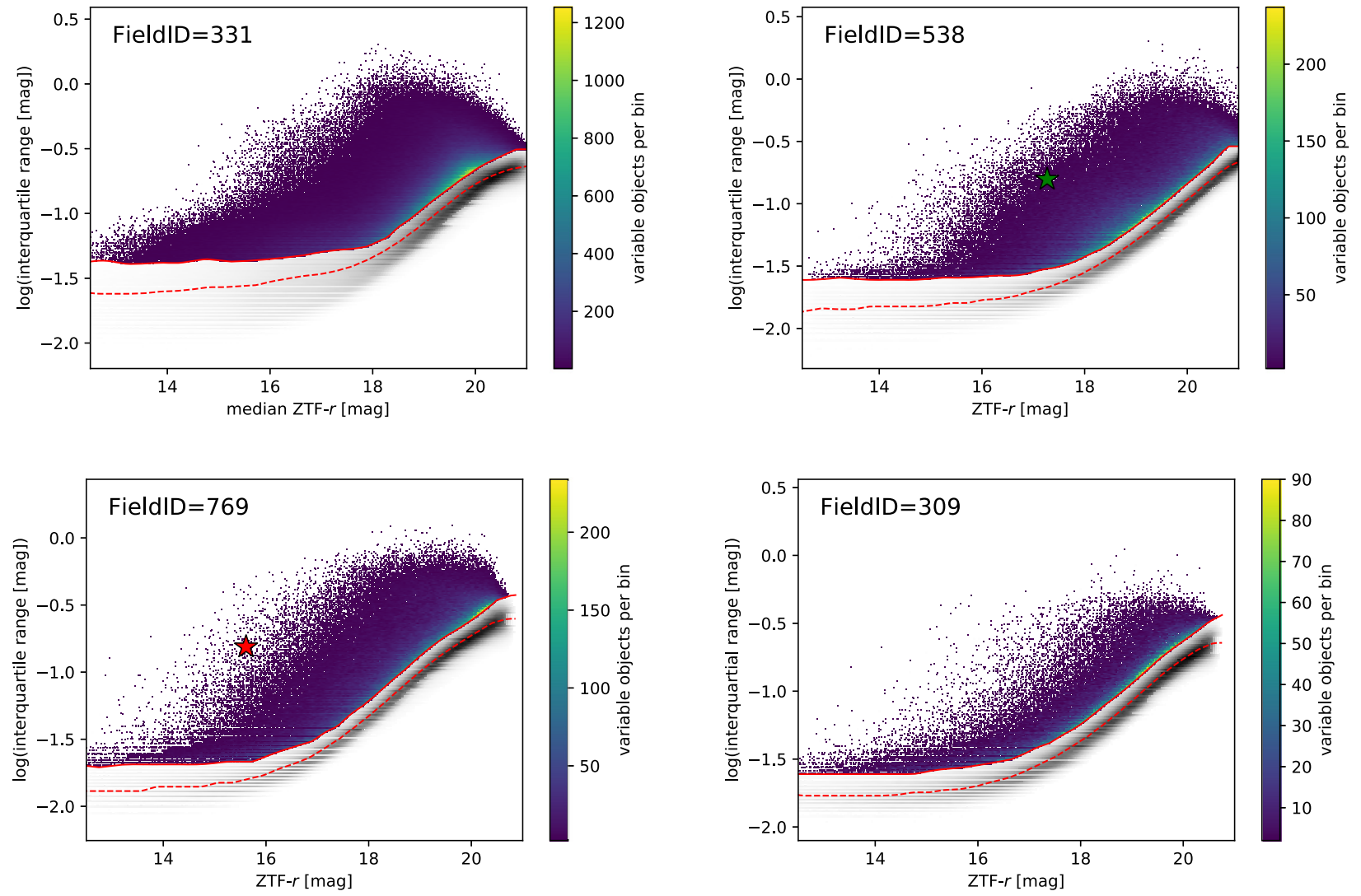

Figure 6. Interquartile range for each field. The grey shaded region corresponds to the underlying distribution of all objects in that field. The colour-coded region corresponds to the objects which are possibly variable. The dashed red line shows the robust median of the underlying grey shaded region. The solid red line corresponds to the limit for potentially variable objects. The red star marks the position of ZTF J2130 (Kupfer et al. 2020a) and the green star marks the position of high-gravity BLAP-3 (Kupfer et al. 2019b).

source which contaminates the same pixel. To test that, we inspected by eye 300 light curves that show the largest IQR for FieldID = 331 which has the highest stellar density and FieldID $=309$ which has the lowest stellar densities of the selected fields. We find that astrophysically variable sources show a trend in the light curve, whereas non-astrophysically variable sources show typically rapid exposure-to-exposure variations. These variations can be explained in most cases by blending. Fig. 7 shows two examples of nonastrophysically variable sources and two examples of astrophysically variable sources. We find that out of the selected light curves only
15 per cent for FieldID $=331$ and 40 per cent for FieldID $=309$ show real astrophysical variability which shows that in the higher density fields the contamination of false positives is significantly larger. This is also consistent with the larger spread in IQR values seen in FieldID = 331 compared to FieldID = 309 (Fig. 5) because a larger number of false positives would lead to a larger spread of IQR values. Combining the variable sources with the fraction of real astrophysical variable sources, we find that $\approx 1-2$ per cent of all sources show significant astrophysical variability in the high-cadence Galactic plane data. We extracted $\approx 230$ million light curves and therefore 

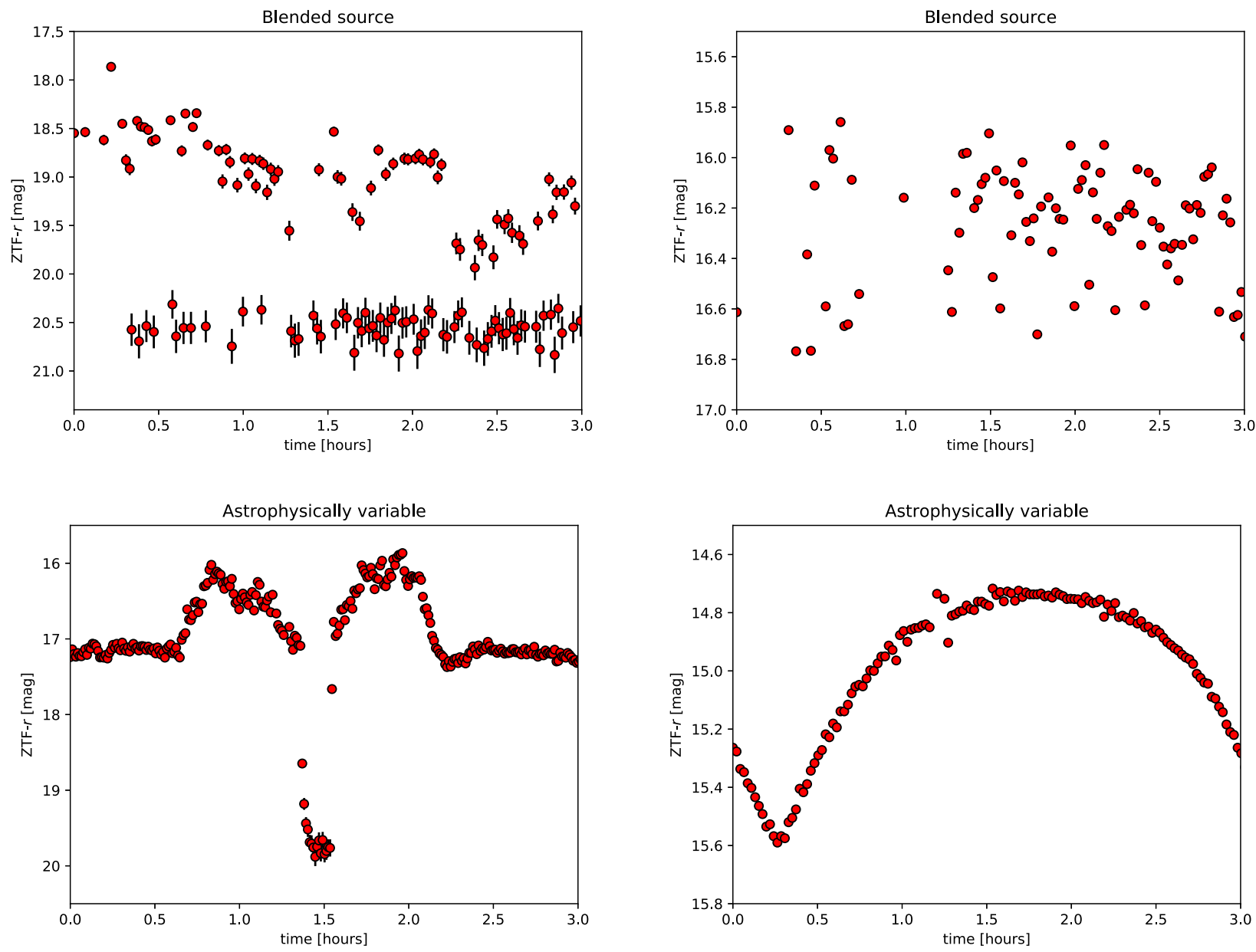

Figure 7. Example light curves of blended sources (upper panels) and real astrophysical variable sources (lower panels).

expect a few million sources which show astrophysical photometric variability during the high-cadence Galactic plane observations.

\section{PROGRESS REPORT}

The primary science driver for the survey is the detection and study of ultracompact binaries and rapid pulsators. Because the data volume is too large to inspect every single light curve, we applied different cuts to pre-select light curves in early analysis. Depending on the science goal different selection criteria are required including a colour cut using PanSTARRS DR2 colours or a selection based on the position in a colour-magnitude diagram using Gaia DR2 (Gaia Collaboration et al. 2016, 2018). In a targeted search for hot subdwarf (sdB/sdO) binaries, we cross-matched the catalogue of 40000 hot subdwarf candidates (Geier et al. 2019) with the ZTF high-cadence Galactic plane data. These searches have already revealed some interesting discoveries, shown in Fig. 8.

As part of our cross-match with the hot subdwarf catalogue, we discovered ZTF J2130, the most compact sdB+WD binary and the first member of systems where the sdB fills its Roche lobe and has started to transfer mass to the WD companion (Upper left-hand panel Fig. 8; $P_{\text {orb }}=39 \mathrm{~min}$; Kupfer et al. 2020a). In a follow-up search, we discovered ZTF J2055, a second member of that class (Kupfer et al. 2020b). Both objects are only a few hundred million years old and members of the Galactic thin disc population, which explains why these objects have not been seen before and shows the value of a high-cadence Galactic plane survey.

Burdge et al. (2020 a,b) presented 12 new ultracompact double white dwarfs including two new AMCVn systems, with orbital periods below $1 \mathrm{~h}$. Although the high-cadence Galactic plane survey only covers $\approx 10$ per cent of the ZTF sky almost half of the new ultracompact double white dwarfs have been covered by the high-cadence Galactic plane observations, including an $8.8 \mathrm{~min}$ binary (Burdge et al. 2020b). Due to the short eclipse duty cycle of $\leq 10$ per cent for some systems, high-cadence Galactic plane observations are ideal to cover enough in-eclipse points to recover the sources in period-finding searches. The upper right-hand panel in Fig. 8 displays an example of an eclipsing double white dwarf binary with an orbital period of $23.7 \mathrm{~min}$ (Burdge et al. 2020a). The lower points every $24 \mathrm{~min}$ indicate an eclipse and shows the short duty cycle of some of the eclipsing double white dwarfs.

The survey revealed a new class of large-amplitude radial-mode compact pulsators (three confirmed objects, middle left-hand panel Fig. 8, Kupfer et al. 2019b). They show typical pulsation amplitudes of 5-20 per cent and periods between 3 and $8 \mathrm{~min}$. Although the stars reside in a region in the Hertzsprung-Russell (HR) diagram usually occupied by helium core burning sdB stars, their pulsation properties are best explained with low-mass pre-He-WDs currently evolving through this part of the HR diagram contracting towards the He-WD cooling tracks. As such they could be the low-mass version of the BLAPs. 

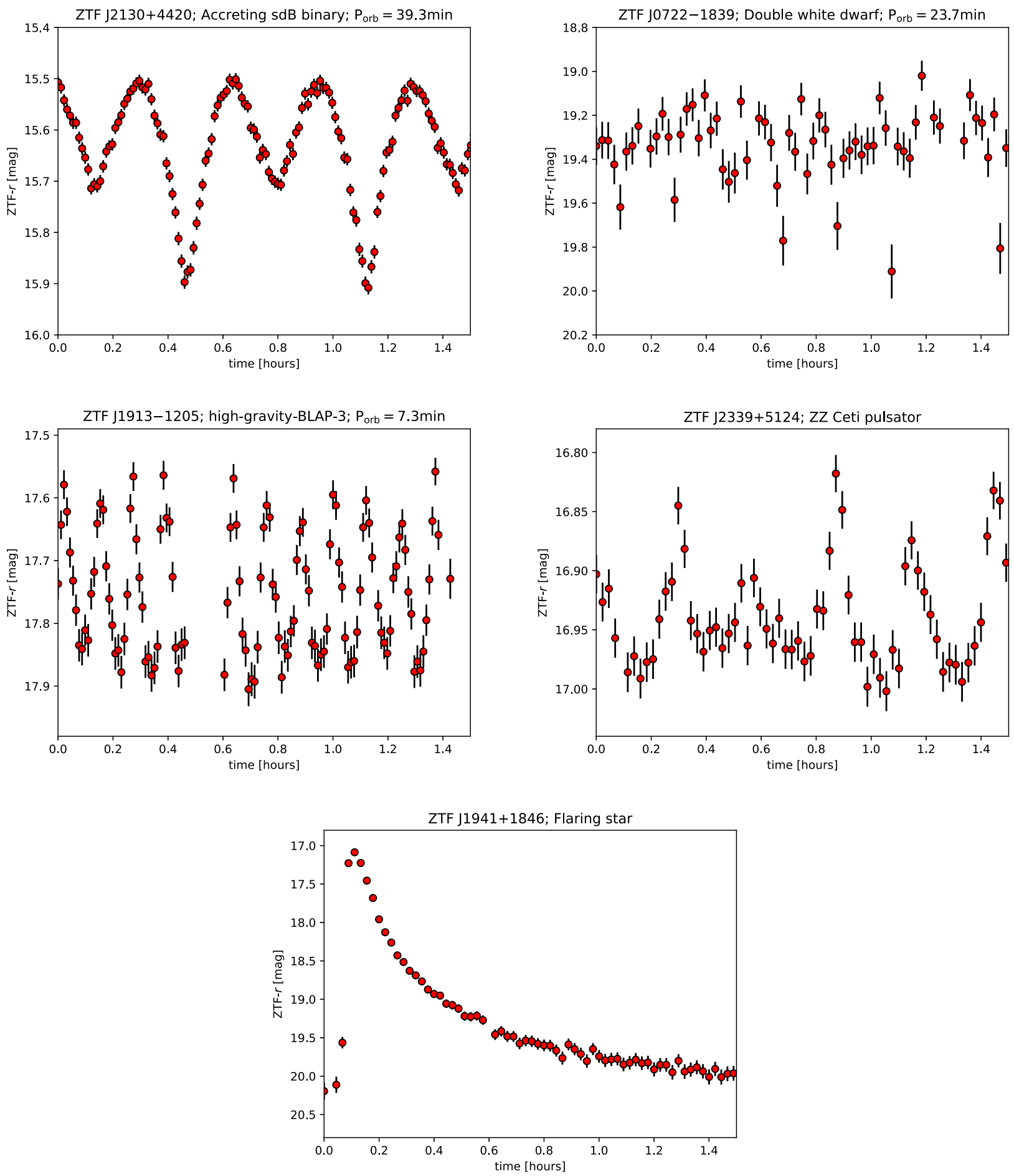

Figure 8. Example light curves of different types of variable objects discovered in the high-cadence Galactic plane survey. Upper left-hand panel: accreting sdB binary (Kupfer et al. 2020a). Upper right-hand panel: eclipsing double white dwarf (Burdge et al. 2020a). Middle left-hand panel: compact radial mode pulsator (Kupfer et al. 2019b). Middle right-hand panel: ZZ Ceti pulsator (Guidry et al. 2020). Lowest panel: flaring star.

The survey furthermore provided the necessary short-cadence observations to resolve the short-period pulsations of ZZ Cetis. These pulsations are evident in the middle right-hand panel of Fig. 8, which highlights ZTF J2339+5124, a new ZZ Ceti identified originally in Guidry et al. (2020). The typical diurnal mean sampling rate of ZTF is too short to resolve the pulsations for many pulsating white dwarfs (ZZ Ceti pulsation modes range from 100-1500 sec Mukadam et al.
2013; Bognár et al. 2020). However, the Galactic plane survey’s 40-s sampling over the span of hours is sufficient to capture consecutive pulsation peaks in ZZ Cetis. These observations may then be coupled with an object's placement relative to the pulsating white dwarf instability strip to allow for its confirmation as a ZZ Ceti (since pulsations are only driven in a narrow range of effective temperature, e.g. Gianninas et al. 2015), as done for ZTF 2339+5124. 
The survey has also provided a large sample of stellar flare detections. These events are the result of rapid magnetic reconnection for low-mass stars, and typically have time-scales of minutes to hours (Hawley et al. 2014), well matched to the cadence of this survey. We can trace flare events in their entirety (e.g. lower panel Fig. 8), measuring precise flare energies when the distance is known, as opposed to flares being detected as single-point outliers in the primary ZTF survey. Using the first data release from the 14 August fields, more than 1500 stellar flare events have been detected (Klein et al., in preparation). This large sample allows us to study correlations between flare occurrence rates and Galactic height.

\section{EARLY RESULTS - NEW SINGLE-MODE HOT SUBDWARF PULSATORS}

Subdwarf B stars (sdBs) are hot stars of spectral type B with luminosities below the main sequence. The formation mechanisms and evolution of $\mathrm{sdBs}$ are still debated, although most $\mathrm{sdBs}$ are thought to be helium (He)-burning stars with masses $\approx 0.5 \mathrm{M}_{\odot}$ and thin hydrogen envelopes (Heber 1986, 2009, 2016). Amongst the sdB stars, two types of multiperiodic pulsators have been discovered, both with generally milli-mag amplitudes. On the hotter side $\left(T_{\text {eff }} \gtrsim\right.$ $28000 \mathrm{~K}$ ) are the V361 Hya stars, which are pressure mode (p-mode) pulsators with typical periods of a few minutes (Kilkenny et al. 1997). On the cooler side, $\left(T_{\text {eff }} \lesssim 28000 \mathrm{~K}\right)$ are the V1093 Her stars which are gravity mode (g-mode) pulsators with periods of $45 \mathrm{~min}$ to $2 \mathrm{~h}$ (Green et al. 2003). Only very few sdBs are know to show amplitudes up to a few percent (Østensen et al. 2010). This includes Balloon 090100001 and PG $1605+072$, which are multiperiodic pulsators with photometric amplitudes of up to 60 mmag (Oreiro et al. 2004; Tillich et al. 2007).

Even before their discovery, the variability of sdBs was predicted to be caused by non-radial pulsation modes driven by the opacity bump due to partial ionization of iron (Charpinet et al. 1996, 1997; Fontaine et al. 2003). Until recently the only known single-mode sdB pulsator was CS 1246, with an amplitude of $\approx 30$ mmag which has decreased below its detection limit over the last few years (Barlow et al. 2010). Barlow et al. (2010) confirmed the object as radial-mode pulsator and found $R=0.19 \pm 0.08 R_{\odot}$ and $M=0.39_{-0.13}^{+0.30} \mathrm{M}_{\odot}$ using the BaadeWesselink method. Pietrukowicz et al. (2017) discovered a new class of radial mode pulsators with large amplitudes up to 300-400 mmag: the BLAPs. More recently a new class of radial mode hot subdwarf B type pulsators (high-gravity BLAPs) was discovered, which show radial mode pulsation amplitudes up 150 mmag (Kupfer et al. 2019b). Unlike most of the multiperiodic sdB pulsators, the BLAP and highgravity BLAPS are more likely to be contracting low-mass helium pre-white dwarfs rather than compact He-core burning stars (Romero et al. 2018; Byrne \& Jeffery 2018, 2020).

We discovered 12 new sdB pulsators that show only a single pulsation mode as part of this survey. We find amplitudes in the ZTF- $r$ band between 20 and 73 mmag which are similar to CS 1246 (Barlow et al. 2010) but smaller than seen in the high-gravity BLAPs (Kupfer et al. 2019b). Each system exhibits only a single frequency in the light curve (see Fig. 9 for the phase folded light curves). The pulsation periods range from 5.8 to $16 \mathrm{~min}$. We performed a colour correction for all objects using 3D extinction maps provided by Green et al. (2019) ${ }^{1}$ and find very similar PanSTARRS colors between $g$ $-r=-0.3$ and $-0.45 \mathrm{mag}$. Using the reddening corrected $g$-band magnitudes and parallaxes from Gaia EDR3 (Gaia Collaboration

${ }^{1}$ http://argonaut.skymaps.info/ et al. 2016, 2020), we find absolute magnitudes in the range $\approx 2.5$ 4.5 mag. Fig. 10 shows their position in a colour-magnitude diagram colour-coded with the pulsation period, which confirms that they are clustered in the region where hot sdBs are located. Table 2 presents an overview of the new pulsators. Remarkably, seven objects of our new sample show very similar pulsation periods around six minutes which is close to the pulsation period of CS 1246, indicating that some of these new pulsators might be related to CS 1246. If they are confirmed as radial-mode pulsators, like CS 1246, future followup observations will measure their masses and radii. Phase-resolved spectroscopy and multiband photometry to fully characterize the objects is underway.

\section{SUMMARY AND CONCLUSIONS}

We have presented an overview and first results of our dedicated high-cadence Galactic plane survey for short-period variable objects, carried out as part of ZTF during its first year of operation. We covered a total of $2990 \mathrm{deg}^{2}$ between 2018 June and January 2019 in ZTF- $r$. Each field was observed continuously at a cadence of $40 \mathrm{~s}$ typically between 1.5 and $3 \mathrm{~h}$ with some fields up 5-6h. We only selected fields with at least 80 epochs and most fields have between 200 and 400 .

The survey covered a total of $\approx 230$ million stars to a limiting magnitude of $20.5 \mathrm{mag}$ in ZTF-r. Stellar densities range from 4 objects per $\operatorname{arcmin}^{2}$ to $\approx 70$ objects per $\operatorname{arcmin}^{2}$. Four fields were selected for a detailed analysis. We calculate light curve statistics for each field and use the IQR to estimate the number of variable objects for each field. We find that $\approx 1-2$ per cent of objects show astrophysical variability in each field which leads to $\approx 2-4$ million expected variable objects in the ZTF Galactic plane high-cadence Galactic plane data. The largest contaminant is blended sources. For the highest density field, this contamination rate is high, with up to 90 per cent of the sources found to be variable from the IQR. This shows that simple light curve statistics can reduce the number of non-variable sources substantially but is not sufficient to select a clean sample in high-density fields. Additional measures are required. In future, instead of using direct (non-difference) images, we will explore the use of forced photometry on difference images using input seed positions provided by external catalogues, e.g. Gaia EDR3. This will provide a more complete census of photometric variability in confused regions of the Galactic plane. Forced photometry is expected to decrease the number of wrong magnitudes due to blending. Additionally, van van Roestel et al. (2021) has shown that machine-learning classification can also substantially decrease the number of false positives.

We have started to explore the data and present a progress report of recently published objects which were discovered by period searches using colour-selected sub-samples. Some of these discoveries revealed new types of variable stars. In this work, we also present a sample of 12 new single-mode hot subdwarf B-star pulsators with pulsation amplitudes between ZTF- $r=20-76 \mathrm{mmag}$ and pulsation periods between $P=5.8-16 \mathrm{~min}$. If they are confirmed as radial mode pulsators future follow-up observations will measure their masses and radii.

Future work is in progress, which applies machine-learning techniques to classify large samples of individual objects (Coughlin et al. 2020; van Roestel et al. 2021). ZTF data up to 2018 December was released in data release 3 and the remaining data obtained in 2019 January was released in data release 4 . ZTF has continued with high-cadence Galactic plane observations in year 2 and 3. We settled on 140 epochs $(1.5 \mathrm{~h}$ ) per field and continued to use the ZTF- $r$ band. 

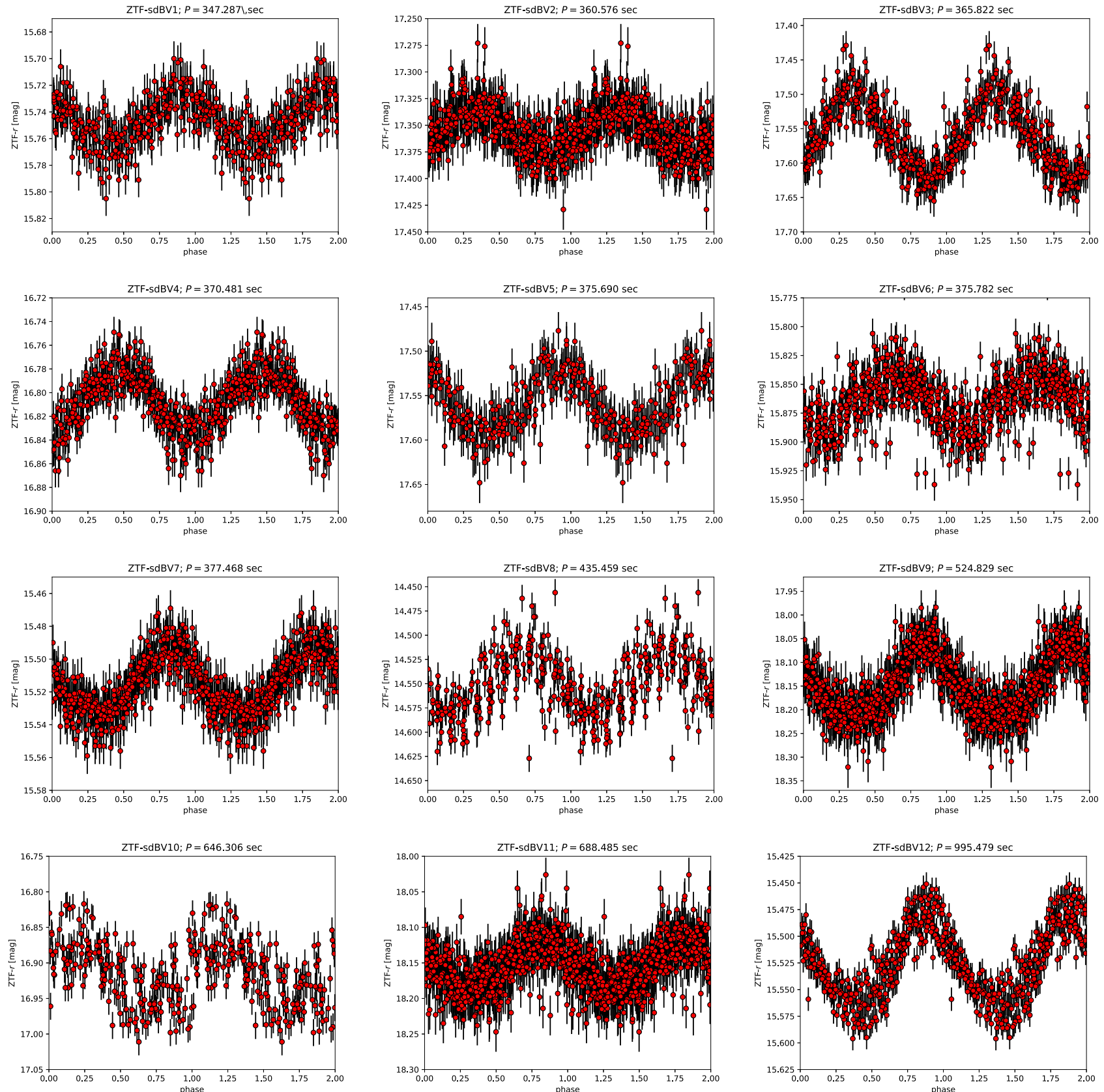

Figure 9. Phase-folded ZTF light curves of the newly discovered radial mode sdB pulsators. All systems were discovered as part of the ZTF high-cadence Galactic plane survey.

We aim to observe the entire Galactic plane within $|b|<10$ and further out to high stellar density regions. We continue to analyse the data using period-finding algorithms and light-curve statistics, but are also exploring the use of neural networks to identify and classify variable stars.

Looking ahead, the Vera Rubin Observatory's Legacy Survey of Space and Time (LSST) will observe to fainter magnitudes and with an expected precision of $\sim 5 \mathrm{mmag}$ is expected to find more variable sources (Huber, Everett \& Howell 2006). Given the significantly larger number of sources in the LSST, the Galactic plane observations of LSST are expected to observe unique sources, which are too faint for ZTF but the high-stellar density will challenge data analysis, and the significantly higher number of sources will require more computational power to search all the objects. 


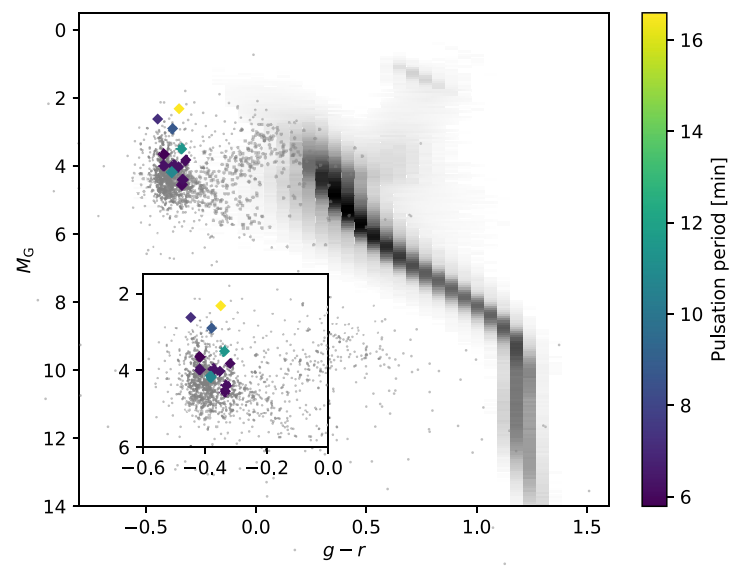

Figure 10. HR diagram of the newly discovered pulsators. The grey dots show known hot subdwarfs selected from Geier (2020) The grey shaded region corresponds to the underlying HR diagram showing the position of the main sequence and the red giant branch.

\section{ACKNOWLEDGEMENTS}

We thank the anonymous referee for their helpful comments and review of the manuscript.

Based on observations obtained with the Samuel Oschin Telescope 48-inch and the 60-inch Telescope at the Palomar Observatory as part of the Zwicky Transient Facility project. ZTF is supported by the National Science Foundation under Grant No. AST-1440341 and a collaboration including Caltech, IPAC, the Weizmann Institute for Science, the Oskar Klein Center at Stockholm University, the University of Maryland, the University of Washington, Deutsches Elektronen-Synchrotron and Humboldt University, Los Alamos National Laboratories, the TANGO Consortium of Taiwan, the University of Wisconsin at Milwaukee, and Lawrence Berkeley National Laboratories. Operations are conducted by COO, IPAC, and UW.

This research was funded by the Gordon and Betty Moore Foundation through Grant GBMF5076. This research was supported in part by the National Science Foundation under Grant No. NSF PHY-1748958.

MWC acknowledges support from the National Science Foundation with grant number PHY-2010970.

This work has made use of data from the European Space Agency (ESA) mission Gaia (https://www.cosmos.esa.int/gaia), processed by the Gaia Data Processing and Analysis Consortium (DPAC, https: //www.cosmos.esa.int/web/gaia/dpac/consortium). Funding for the DPAC has been provided by national institutions, in particular, the institutions participating in the Gaia Multilateral Agreement.

\section{DATA AVAILABILITY}

All the observational data used in this paper are publicly available at the IRSA data base and can be accessed here: https://www.ztf.caltec h.edu/page/dr4.

\section{REFERENCES}

Baker J. et al., 2019, Bull. Am. Astron. Soc., 51, 123

Barclay T., Ramsay G., Hakala P., Napiwotzki R., Nelemans G., Potter S., Todd I., 2011, MNRAS, 413, 2696

Barlow B. N. et al., 2010, MNRAS, 403, 324

Bellm E. C. et al., 2019a, Publ. Astron. Soc. Pac., 131, 018002
Bellm E. C. et al., 2019b, Publ. Astron. Soc. Pac., 131, 068003

Bertin E., Arnouts S., 1996, A\&AS, 117, 393

Bognár Z. et al., 2020, A\&A, 638, A82

Breger M., 2000, in Breger M., Montgomery M., eds, ASP Conf. Ser., Vol. 210, Delta Scuti and Related Stars. Astron. Soc. Pac., San Francisco, p. 3

Burdge K. B. et al., 2019, Nature, 571, 528

Burdge K. B. et al., 2020a, ApJ, 905, 32

Burdge K. B. et al., 2020b, ApJ, 905, L7

Byrne C. M., Jeffery C. S., 2018, MNRAS, 481, 3810

Byrne C. M., Jeffery C. S., 2020, MNRAS, 492, 232

Chambers K. C. et al., 2016, preprint (arXiv:e-print)

Charpinet S., Fontaine G., Brassard P., Chayer P., Rogers F. J., Iglesias C. A., Dorman B., 1997, ApJ, 483, L123

Charpinet S., Fontaine G., Brassard P., Dorman B., 1996, ApJ, 471, L103

Coughlin M. W. et al., 2020, preprint (arXiv:2009.14071)

Fontaine G., Brassard P., 2008, Publ. Astron. Soc. Pac., 120, 1043

Fontaine G., Brassard P., Charpinet S., Green E. M., Chayer P., Billères M., Randall S. K., 2003, ApJ, 597, 518

Gaia Collaboration, Brown A. G. A., Vallenari A., Prusti T., de Bruijne J. H. J., Babusiaux C., Biermann M., 2020, A\&A, 649, 20

Gaia Collaboration et al., 2016, A\&A, 595, A1

Gaia Collaboration et al., 2018, A\&A, 616, A1

Geier S., 2020, A\&A, 635, A193

Geier S., Raddi R., Gentile Fusillo N. P., Marsh T. R., 2019, A\&A, 621, A38

Gianninas A., Kilic M., Brown W. R., Canton P., Kenyon S. J., 2015, ApJ, 812,167

Graham M. J. et al., 2019, PASP, 131, 078001

Green E. M. et al., 2003, Ap\&SS, 284, 65

Green G. M., Schlafly E., Zucker C., Speagle J. S., Finkbeiner D., 2019, ApJ, 887,93

Guidry J. A. et al., 2020, ApJ, 912, 24

Hawley S. L., Davenport J. R. A., Kowalski A. F., Wisniewski J. P., Hebb L., Deitrick R., Hilton E. J., 2014, ApJ, 797, 121

Heber U., 1986, A\&A, 155, 33

Heber U., 2009, ARA\&A, 47, 211

Heber U., 2016, Publ. Astron. Soc. Pac., 128, 082001

Heinze A. N. et al., 2018, AJ, 156, 241

Hermes J. J. et al., 2013, ApJ, 765, 102

Huber M. E., Everett M. E., Howell S. B., 2006, AJ, 132, 633-649

Jayasinghe T. et al., 2018, MNRAS, 477, 3145

Kilkenny D., Koen C., O’Donoghue D., Stobie R. S., 1997, MNRAS, 285, 640

Korol V., Rossi E. M., Groot P. J., Nelemans G., Toonen S., Brown A. G. A., 2017, MNRAS, 470, 1894

Kremer K., Breivik K., Larson S. L., Kalogera V., 2017, ApJ, 846, 95

Kupfer T., Kilic M., Maccarone T., Burns E., Fryer C. L., Wilson-Hodge C. A., 2019a, Bull. Am. Astron. Soc., 51, 188

Kupfer T. et al., 2017, ApJ, 851, 28

Kupfer T. et al., 2018, MNRAS, 480, 302

Kupfer T. et al., 2019b, ApJ, 878, L35

Kupfer T. et al., 2020a, ApJ, 891, 45

Kupfer T. et al., 2020b, ApJ, 898, L25

Lamberts A., Blunt S., Littenberg T. B., Garrison-Kimmel S., Kupfer T., Sanderson R. E., 2019, MNRAS, 490, 5888

Law N. M. et al., 2009, Publ. Astron. Soc. Pac., 121, 1395

Littenberg T. B., Larson S. L., Nelemans G., Cornish N. J., 2013, MNRAS, 429,2361

Littenberg T. et al., 2019, Bull. Am. Astron. Soc., 51, 34

Macfarlane S. A., Toma R., Ramsay G., Groot P. J., Woudt P. A., Drew J. E., Barentsen G., Eislöffel J., 2015, MNRAS, 454, 507

Macfarlane S. A. et al., 2017a, MNRAS, 465, 434

Macfarlane S. A. et al., 2017b, MNRAS, 470, 732

Masci F. J. et al., 2019, Publ. Astron. Soc. Pac., 131, 018003

Meng X.-C., Han Z.-W., Podsiadlowski P., Li J., 2020, ApJ, 903, 100

Mukadam A. S. et al., 2013, ApJ, 771, 17

Nelemans G., Yungelson L. R., Portegies Zwart S. F., 2004, MNRAS, 349, 181 
Nemec J., Mateo M., 1990, in Cacciari C., Clementini G., eds, ASP Conf. Ser., Vol. 11, Confrontation Between Stellar Pulsation and Evolution. Astron. Soc. Pac., San Francisco, p. 64

Nissanke S., Vallisneri M., Nelemans G., Prince T. A., 2012, ApJ, 758, 131 Oreiro R., Ulla A., Pérez Hernández F., Østensen R., Rodríguez López C., MacDonald J., 2004, A\&A, 418, 243

Østensen R. H. et al., 2010, MNRAS, 409, 1470

Pietrukowicz P. et al., 2017, Nat. Astron., 1, 0166

Ramsay G., Hakala P., 2005, MNRAS, 360, 314

Renson P., Gerbaldi M., Catalano F. A., 1991, A\&AS, 89, 429

Romero A. D., Córsico A. H., Althaus L. G., Pelisoli I., Kepler S. O., 2018, MNRAS, 477, L30

Saito R. K. et al., 2012, A\&A, 537, A107

Shah S., Nelemans G., 2014, ApJ, 790, 161

Shah S., van der Sluys M., Nelemans G., 2012, A\&A, 544, A153

Shappee B. J. et al., 2014, ApJ, 788, 48
Soszyński I. et al., 2015, AcA, 65, 39

Stetson P. B., 1987, Publ. Astron. Soc. Pac., 99, 191

Ströer A., Vecchio A., 2006, Class. Quantum Gravity, 23, 809

Tillich A., Heber U., O’Toole S. J., Østensen R., Schuh S., 2007, A\&A, 473, 219

Toma R. et al., 2016, MNRAS, 463, 1099

Tonry J. L. et al., 2018, Publ. Astron. Soc. Pac., 130, 064505

van Roestel J. et al., 2019, Res. Notes Am. Astron. Soc., 3, 136

van Roestel J. et al., 2021, ApJ, 161, 17

York D. G. et al., 2000, AJ, 120, 1579

\section{APPENDIX A: FIELDS OBSERVED AS PART OF THE HIGH-CADENCE GALACTIC PLANE OBSERVATIONS IN ZTF YEAR-1}

Table A1. Fields observed as part of the June/July campaign. Median values are given for limiting magnitude and seeing.

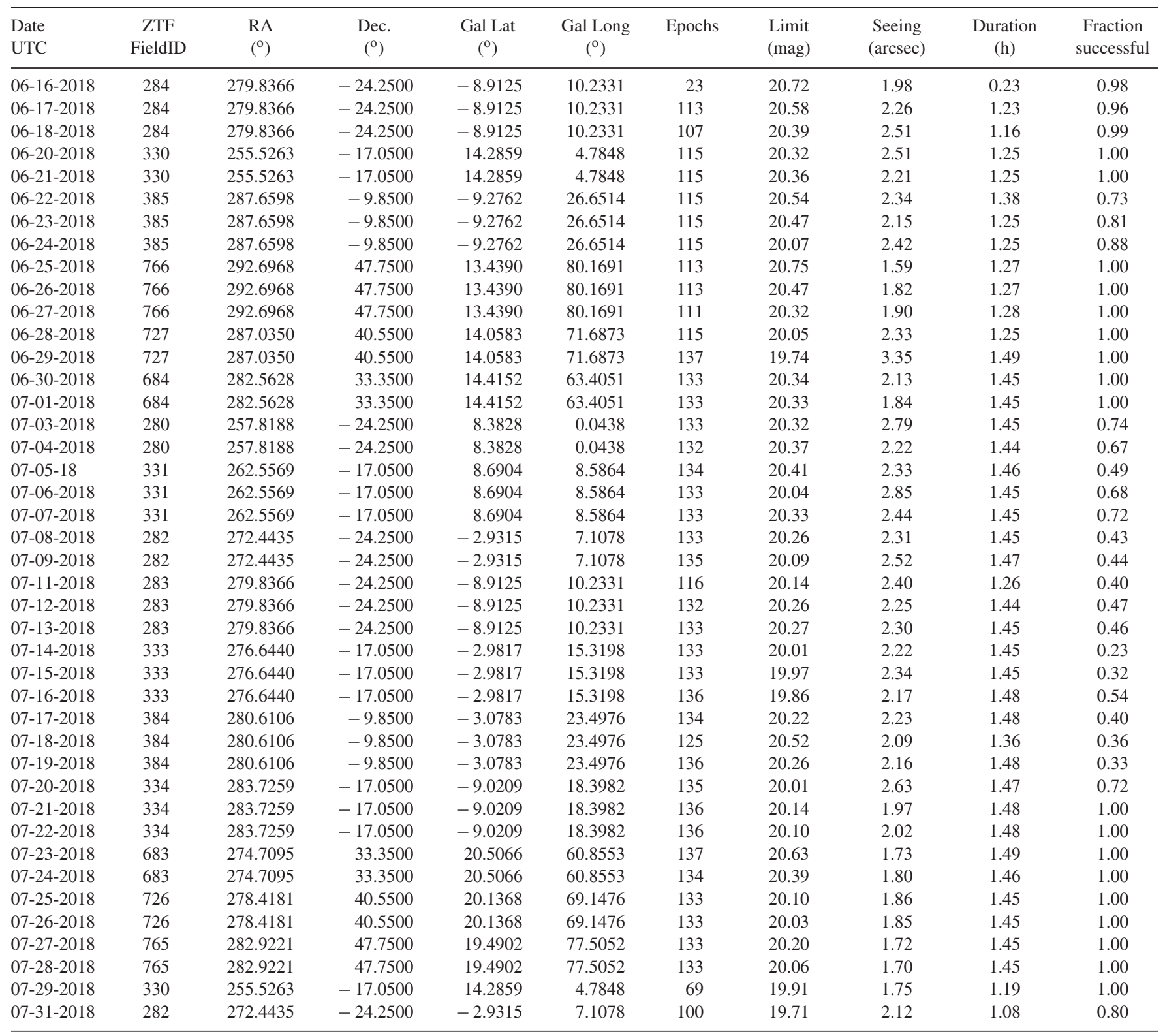


Table A2. Fields observed as part of the August campaign. Median values are given for limiting magnitude and seeing.

\begin{tabular}{|c|c|c|c|c|c|c|c|c|c|c|}
\hline $\begin{array}{l}\text { Date } \\
\text { UTC }\end{array}$ & $\begin{array}{c}\text { ZTF } \\
\text { FieldID }\end{array}$ & $\begin{array}{l}\mathrm{RA} \\
\left({ }^{\circ}\right)\end{array}$ & $\begin{array}{c}\text { Dec. } \\
\left({ }^{\circ}\right)\end{array}$ & $\begin{array}{c}\text { Gal Lat } \\
\left(^{\circ}\right)\end{array}$ & $\begin{array}{c}\text { Gal Long } \\
\left({ }^{\circ}\right)\end{array}$ & Epochs & $\begin{array}{l}\text { Limit } \\
(\mathrm{mag})\end{array}$ & $\begin{array}{l}\text { Seeing } \\
(\operatorname{arcsec})\end{array}$ & $\begin{array}{c}\text { Duration } \\
\text { (h) }\end{array}$ & $\begin{array}{l}\text { Fraction } \\
\text { successful }\end{array}$ \\
\hline 08-03-2018 & 538 & 284.8764 & 11.7500 & 3.0889 & 44.6385 & 118 & 20.84 & 1.87 & 1.28 & 1.00 \\
\hline 08-03-2018 & 539 & 277.9013 & 11.7500 & 9.1851 & 41.5422 & 42 & 20.66 & 1.97 & 0.45 & 1.00 \\
\hline 08-04-2018 & 538 & 284.8764 & 11.7500 & 3.0889 & 44.6385 & 118 & 20.93 & 1.87 & 2.59 & 1.00 \\
\hline 08-04-2018 & 539 & 277.9013 & 11.7500 & 9.1851 & 41.5422 & 118 & 20.76 & 2.19 & 2.59 & 1.00 \\
\hline 08-07-2018 & 638 & 285.7714 & 26.1500 & 2.9069 & 61.0817 & 118 & 21.09 & 1.89 & 2.67 & 1.00 \\
\hline 08-07-2018 & 639 & 293.2531 & 26.1500 & -2.8742 & 64.5379 & 118 & 20.97 & 2.08 & 2.67 & 1.00 \\
\hline 08-08-2018 & 638 & 285.7714 & 26.1500 & 2.9069 & 61.0817 & 118 & 21.03 & 1.84 & 2.67 & 1.00 \\
\hline 08-08-2018 & 639 & 293.2531 & 26.1500 & -2.8742 & 64.5379 & 118 & 20.90 & 2.03 & 2.67 & 1.00 \\
\hline 08-10-2018 & 488 & 288.1194 & 4.5500 & -3.0953 & 39.7486 & 118 & 20.55 & 2.01 & 2.59 & 1.00 \\
\hline 08-10-2018 & 489 & 295.1303 & 4.5500 & -9.2520 & 43.0768 & 118 & 20.42 & 2.14 & 2.59 & 1.00 \\
\hline 08-11-2018 & 540 & 291.9311 & 11.7500 & -3.0068 & 47.8872 & 54 & 20.52 & 2.00 & 2.31 & 1.00 \\
\hline 08-11-2018 & 541 & 298.9153 & 11.7500 & -8.9492 & 51.2901 & 55 & 20.54 & 2.04 & 1.26 & 1.00 \\
\hline 08-12-2018 & 540 & 291.9311 & 11.7500 & -3.0068 & 47.8872 & 117 & 20.67 & 2.12 & 2.57 & 1.00 \\
\hline 08-12-2018 & 541 & 298.9153 & 11.7500 & -8.9492 & 51.2901 & 116 & 20.57 & 2.22 & 2.55 & 1.00 \\
\hline 08-13-2018 & 591 & 296.1269 & 18.9500 & -2.9775 & 56.1558 & 118 & 20.80 & 2.09 & 2.61 & 1.00 \\
\hline 08-13-2018 & 592 & 303.3767 & 18.9500 & -8.8128 & 59.7765 & 118 & 20.85 & 2.01 & 2.61 & 1.00 \\
\hline 08-14-2018 & 591 & 296.1269 & 18.9500 & -2.9775 & 56.1558 & 118 & 20.92 & 1.87 & 2.63 & 1.00 \\
\hline 08-14-2018 & 592 & 303.3767 & 18.9500 & -8.8128 & 59.7765 & 118 & 20.95 & 1.95 & 2.63 & 1.00 \\
\hline 08-15-2018 & 685 & 290.4160 & 33.3500 & 8.4910 & 66.2776 & 117 & 20.92 & 1.95 & 2.77 & 1.00 \\
\hline $08-15-2018$ & 686 & 298.2693 & 33.3500 & 2.7706 & 69.5025 & 117 & 20.77 & 2.09 & 2.77 & 1.00 \\
\hline 08-16-2018 & 685 & 290.4160 & 33.3500 & 8.4910 & 66.2776 & 112 & 20.93 & 1.81 & 2.81 & 0.85 \\
\hline 08-16-2018 & 686 & 298.2693 & 33.3500 & 2.7706 & 69.5025 & 111 & 20.81 & 1.95 & 2.79 & 0.86 \\
\hline 08-17-2018 & 436 & 284.3894 & -2.65000 & -3.1176 & 31.6292 & 97 & 20.37 & 2.19 & 2.79 & 0.98 \\
\hline 08-17-2018 & 437 & 291.4182 & -2.65000 & -9.3570 & 34.8701 & 94 & 20.47 & 2.12 & 2.79 & 1.00 \\
\hline 08-18-2018 & 436 & 284.3894 & -2.65000 & -3.1176 & 31.6292 & 118 & 20.51 & 2.16 & 2.57 & 0.97 \\
\hline 08-18-2018 & 437 & 291.4182 & -2.65000 & -9.3570 & 34.8701 & 118 & 20.61 & 2.04 & 2.57 & 1.00 \\
\hline
\end{tabular}


Table A3. Fields observed as part of the winter campaign. Median values are given for limiting magnitude and seeing.

\begin{tabular}{|c|c|c|c|c|c|c|c|c|c|c|}
\hline $\begin{array}{l}\text { Date } \\
\text { UTC }\end{array}$ & $\begin{array}{c}\text { ZTF } \\
\text { FieldID }\end{array}$ & $\begin{array}{l}\text { RA } \\
\left({ }^{\circ}\right)\end{array}$ & $\begin{array}{c}\text { Dec. } \\
\left({ }^{\circ}\right)\end{array}$ & $\begin{array}{l}\text { Gal Lat } \\
\left({ }^{\circ}\right)\end{array}$ & $\begin{array}{l}\text { Gal Long } \\
\left({ }^{\circ}\right)\end{array}$ & Epochs & $\begin{array}{l}\text { Limit } \\
(\mathrm{mag})\end{array}$ & $\begin{array}{c}\text { Seeing } \\
(\operatorname{arcsec})\end{array}$ & $\begin{array}{c}\text { Duration } \\
\text { (hrs) }\end{array}$ & $\begin{array}{c}\text { Fraction } \\
\text { successful }\end{array}$ \\
\hline $11-15-2018$ & 728 & 295.6518 & 40.5500 & 8.2113 & 74.6857 & 148 & 19.93 & 2.81 & 3.29 & 1.00 \\
\hline $11-15-2018$ & 729 & 304.2686 & 40.5500 & 2.6520 & 78.1565 & 148 & 20.04 & 2.74 & 3.29 & 1.00 \\
\hline 11-19-2018 & 799 & 291.0910 & 54.9500 & 17.3604 & 86.5193 & 145 & 20.16 & 1.72 & 3.32 & 0.92 \\
\hline $11-19-2018$ & 800 & 302.1884 & 54.9500 & 11.7051 & 89.5415 & 146 & 20.08 & 1.74 & 3.90 & 0.90 \\
\hline $11-26-2018$ & 767 & 302.4714 & 47.7500 & 7.7191 & 83.4543 & 128 & 20.30 & 1.81 & 2.88 & 1.00 \\
\hline $11-26-2018$ & 768 & 312.2461 & 47.7500 & 2.4194 & 87.3502 & 128 & 20.42 & 1.81 & 2.88 & 1.00 \\
\hline $11-28-2018$ & 688 & 313.8717 & 33.3500 & -7.8072 & 77.1136 & 142 & 19.66 & 1.91 & 3.75 & 0.80 \\
\hline $11-28-2018$ & 689 & 321.6554 & 33.3500 & -12.5790 & 81.6002 & 142 & 19.78 & 1.87 & 3.76 & 0.86 \\
\hline $11-29-2018$ & 767 & 302.4714 & 47.7500 & 7.7191 & 83.4543 & 127 & 20.62 & 1.81 & 2.86 & 1.00 \\
\hline $11-29-2018$ & 768 & 312.2461 & 47.7500 & 2.4194 & 87.3502 & 133 & 20.67 & 1.80 & 2.86 & 1.00 \\
\hline $12-03-2018$ & 730 & 312.8218 & 40.5500 & -2.5179 & 82.0919 & 59 & 20.62 & 1.81 & 1.30 & 1.00 \\
\hline $12-03-2018$ & 731 & 321.3116 & 40.5500 & -7.2339 & 86.5070 & 109 & 20.67 & 1.80 & 1.84 & 0.98 \\
\hline $12-05-2018$ & 412 & 114.2600 & -2.6500 & 9.3964 & 220.9648 & 275 & 20.39 & 2.46 & 6.01 & 1.00 \\
\hline $12-09-2018$ & 770 & 332.1016 & 47.7500 & -6.6404 & 97.1225 & 134 & 20.24 & 2.35 & 3.97 & 1.00 \\
\hline $12-09-2018$ & 771 & 342.1387 & 47.7500 & -10.1214 & 102.9618 & 132 & 20.42 & 2.26 & 3.95 & 1.00 \\
\hline $12-09-2018$ & 259 & 104.6634 & -24.2500 & -8.9125 & 235.7669 & 111 & 19.77 & 2.19 & 1.26 & 0.95 \\
\hline $12-12-2018$ & 308 & 100.7741 & -17.0500 & -9.0209 & 227.6018 & 140 & 20.32 & 2.53 & 3.05 & 0.97 \\
\hline $12-12-2018$ & 309 & 107.8560 & -17.0500 & -2.9817 & 230.6802 & 140 & 20.26 & 2.66 & 3.05 & 1.00 \\
\hline $12-13-2018$ & 308 & 100.7741 & -17.0500 & -9.0209 & 227.6018 & 78 & 19.96 & 3.16 & 1.69 & 1.00 \\
\hline $12-13-2018$ & 309 & 107.8560 & -17.0500 & -2.9817 & 230.6802 & 78 & 19.99 & 3.14 & 1.69 & 1.00 \\
\hline $12-13-2018$ & 769 & 322.0645 & 47.7500 & -2.3827 & 91.8751 & 269 & 19.83 & 3.35 & 2.94 & 1.00 \\
\hline $12-14-2018$ & 769 & 322.0645 & 47.7500 & -2.3827 & 91.8751 & 270 & 20.06 & 2.36 & 2.95 & 0.92 \\
\hline $12-16-2018$ & 803 & 335.1841 & 54.9500 & -1.7248 & 102.7388 & 345 & 20.21 & 2.11 & 3.77 & 1.00 \\
\hline $12-16-2018$ & 257 & 89.8772 & -24.2500 & -21.2513 & 230.1444 & 198 & 20.42 & 2.02 & 2.16 & 1.00 \\
\hline $12-24-2018$ & 804 & 345.7743 & 54.9500 & -4.5684 & 108.1171 & 153 & 20.06 & 1.75 & 3.49 & 0.94 \\
\hline $12-24-2018$ & 805 & 356.3646 & 54.9500 & -6.5258 & 113.8966 & 153 & 20.17 & 1.71 & 3.49 & 0.95 \\
\hline $12-24-2018$ & 260 & 112.0564 & -24.2500 & -2.9315 & 238.8922 & 189 & 19.52 & 2.10 & 2.06 & 0.81 \\
\hline $12-28-2018$ & 804 & 345.7743 & 54.9500 & -4.5684 & 108.1171 & 58 & 19.60 & 3.87 & 1.31 & 0.82 \\
\hline $12-28-2018$ & 805 & 356.3646 & 54.9500 & -6.5258 & 113.8966 & 87 & 19.83 & 3.57 & 1.28 & 0.88 \\
\hline $12-28-2018$ & 260 & 112.0564 & -24.2500 & -2.9315 & 238.8922 & 196 & 19.44 & 3.83 & 2.14 & 0.94 \\
\hline $12-29-2018$ & 806 & 6.6666 & 62.1500 & -0.3373 & 120.3949 & 87 & 19.86 & 3.84 & 0.84 & 1.00 \\
\hline $12-29-2018$ & 260 & 112.0564 & -24.2500 & -2.9315 & 238.8922 & 196 & 19.75 & 3.25 & 2.14 & 1.00 \\
\hline $12-30-2018$ & 462 & 110.1486 & 4.5500 & 9.0982 & 212.5713 & 275 & 20.09 & 2.91 & 6.01 & 0.98 \\
\hline $12-30-2018$ & 463 & 116.9904 & 4.5500 & 15.1705 & 215.7493 & 274 & 20.09 & 2.96 & 5.99 & 0.98 \\
\hline 01-03-2019 & 261 & 119.3957 & -24.2500 & 2.8444 & 242.2711 & 172 & 19.55 & 4.05 & 1.87 & 0.93 \\
\hline $01-04-2019$ & 261 & 119.3957 & -24.2500 & 2.8444 & 242.2711 & 171 & 20.00 & 2.83 & 1.83 & 1.00 \\
\hline 01-04-2019 & 258 & 97.2703 & -24.2500 & -15.0272 & 232.8673 & 163 & 20.22 & 2.60 & 1.78 & 1.00 \\
\hline 01-08-2019 & 360 & 110.9488 & -9.8500 & 3.0707 & 225.7543 & 333 & 19.91 & 3.57 & 3.64 & 0.91 \\
\hline 01-10-2019 & 262 & 126.6811 & -24.2500 & 8.3828 & 245.9562 & 172 & 20.32 & 2.29 & 1.87 & 0.95 \\
\hline 01-10-2019 & 260 & 112.0564 & -24.2500 & -2.9315 & 238.8922 & 159 & 20.13 & 2.58 & 1.73 & 1.00 \\
\hline 01-11-2019 & 311 & 121.9430 & -17.0500 & 8.6904 & 237.4136 & 275 & 20.58 & 2.32 & 3.00 & 1.00 \\
\hline
\end{tabular}

This paper has been typeset from a $\mathrm{T}_{\mathrm{E}} \mathrm{X} / \mathrm{LAT} \mathrm{E} \mathrm{X}$ file prepared by the author. 